\title{
Estudo epidemiológico da dacriocistite crônica
}

\author{
Epidemiological study of chronic dacryocystitis
}

Silvia Helena Tavares Lorena1 ${ }^{1}$ João Amaro Ferrari Silva²

\section{ResUMo}

Objetivo: O objetivo deste projeto de pesquisa é documentar as características epidemiológicas dos casos de dacriocistites crônicas atendidos em um hospital público. Métodos: Estudo retrospectivo e documental dos prontuários de 60 pacientes com história pregressa de dacriocistite crônica que foram atendidos, no período de 12 de janeiro de 2009 a 9 de junho de 2010. Os pacientes, no período em estudo, foram submetidos à avaliação oftalmológica: inspeção ocular, biomicroscopia,Teste de Milder e Teste de Jones I e II. Resultados: A média de idade dos pacientes com dacriocistite crônica atendidos foi de 55 anos, sendo 73 \% (44) do sexo feminino e 27 \% (16) do sexo masculino. O envolvimento unilateral ocorreu em 95\% (57) dos casos de dacriocistite crônica e somente 5 \% (3) foram bilaterais.. Neste trabalho, o acometimento do lado direito foi de $45 \%$ (26) e do lado esquerdo $55 \%$ (31) respectivamente. Em relação à raça, prevaleceu a branca com 92\% (54) dos casos de dacriocistite crônica e 8\% (6) da raça negra. A evolução para dacriocistite aguda ocorreu em $20 \%$ dos pacientes. Em relação à etiologia da dacriocistite crônica, houve predominância da idiopática em 95\% ( 57) dos casos e somente 5\% (3) devido a trauma. Conclusão: A dacriocistite é mais frequente nas mulheres, rara na raça negra e apresenta predisposição hereditária, aumentando a incidência a partir da $6^{\text {a década. }}$

Descritores:Dacriocistite crônica/epidemiologia; Obstrução dos ductos lacrimais; Adulto

\begin{abstract}
Purpose:The goal of this project is to document the epidemiological characteristics of chronic dacryocystitis cases assisted in a public hospital. Methods: This is a retrospective study of medical records of patients who developed chronic dacryocystitis assisted in the period of january $12^{\text {th }}, 2009$ to june $9^{\text {th }}, 2010$. The patients were ophthalmologist evaluated: ocular inspection, biomicroscopy, Milder Test, Test of Jones I and II. Results: The gender with chronic dacryocystitis belonged to 55 years. There were $73 \%$ female and $27 \%$ male. The unilateral involvement occurred in $95 \%$ of the cases and only $5 \%$ were bilateral. In this work, the side of this pathology was on the right in $45 \%$ and on the left in $55 \%$. Negroid races have a lower rate of illness than caucasians.Regarding the chronic dacryocystitis ethiology, there was a predominance of the idiopathic in $95 \%$ of the cases and only $5 \%$ of trauma. Conclusion: The dacryocystitis is more frequent in women, rare in blacks and presents hereditary predisposition, increasing the incidence from the 6th decade
\end{abstract}

Keywords: Chronic dacryocystitis/epidemiology; Lacrimal duct obstruction;Adult

\footnotetext{
'Médica Oftalmologista Colaboradora, Pós-graduanda nível Doutorado do Setor de Vias Lacrimais da Universidade Federal de São Paulo - (UNIFESP) - São Paulo (SP), Brasil;

${ }^{2}$ Chefe do Setor de Vias Lacrimais da Universidade Federal de São Paulo - (UNIFESP) - São Paulo (SP), Brasil.
}

Trabalho realizado no Setor de Vias Lacrimais da Universidade Federal de São Paulo - (UNIFESP - São Paulo (SP), Brasil.

Os autores declaram inexistir conflitos de interesse

Recebido para publicação em: 20/10/2010 - Aceito para publicação em 20/7/2011

Rev Bras Oftalmol. 2011; 70 (6): 396-9 


\section{INTRODUÇÃO}

A inflamação do saco lacrimal (dacriocistite) resulta da estase da lágrima, devido uma obstrução do ducto lácrimonasal, propiciando a proliferação de bactérias ${ }^{(1)}$.

A obstrução do ducto lácrimonasal pode ser congênita em $2 \%$ a $6 \%$ dos casos ou adquirida ${ }^{(2,3)}$.

A causa da obstrução pode ser primária ou secundária. A causa primária refere-se à etiologia idiopática, enquanto que a causa secundária é devido à sinusite, trauma naso-orbital, doenças sistêmicas, neoplasia ou secundária à obstrução congênita não curada.

A incidência da dacriocistite crônica é de $2 \%$ a $3 \%$ em relação aos outros tipos de obstrução da via lacrimal de drenagem ${ }^{(4,5)}$.

Conforme a literatura ${ }^{(5)}$, é recomendada a biopsia do saco lacrimal em pacientes submetidos à dacriocistorrinostomia que possuam antecedentes de doença sistêmica ou uma aparência anormal do saco lacrimal durante a cirurgia.

A dacriocistite é mais frequente nas mulheres, rara na raça negra e apresenta predisposição hereditária ${ }^{(6)}$. Manifesta-se com epífora, às vezes, com conjuntivite crônica ou recorrente, ocasionando edema indolor no canto medial, abaixo da ligamento palpebral medial. Uma vez que a secreção purulenta acumula no interior do saco lacrimal, ocasiona uma ectasia se manifestando como um abaulamento cístico sob a pele.Uma compressão na região do saco lacrimal resulta em refluxo de material purulento para o fórnice conjuntival.

O paciente se queixa de turvação visual devido ao aumento da espessura do filme lacrimal.

Os agentes etiológicos mais frequentes desta patologia são: Staphylococcus aureus e Staphylococcus epidermitis $^{(7,8)}$.

Em relação ao tratamento, se recomenda medidas de higiene associadas com colírios antissépticos e antibióticos. O tratamento definitivo é o cirúrgico (dacriocistorrinostomia externa ou endonasal)

O diagnóstico diferencial da dacriocistite se faz com: abscessos cutâneos etmoidite anterior aguda, dermóides, hemangiomas cavernoso, fibromas, dacriolitíase e mucocele de saco lacrimal ${ }^{(8,9)}$.

O objetivo deste estudo é a análise epidemiológica da dacriocistite crônica em relação ao sexo, idade e lateralidade.

\section{Métodos}

Estudo retrospectivo e documental dos prontuários de 60 pacientes com história pregressa de dacriocistite crônica que foram atendidos no Ambulatório de Vias Lacrimais da UNIFESP, no período de 12 de janeiro de 2009 a 9 de junho de 2010 .

Os pacientes, no período em estudo, foram submetidos à avaliação oftalmológica: inspeção ocular, biomicroscopia, Teste de Milder e Teste de Jones I e II.

A inspeção ocular foi realizada com a finalidade de diagnosticar as más posições palpebrais (entrópio, ectrópio, paralisia facial) e na presença de massa, no canto medial ocular, verificar as suas características (mole, dura, retrátil, pulsátil e a presença de refluxo de secreção para o fórnice conjuntival).

A biomicroscopia foi realizada, a fim de detectar uma pseudoepífora, que consiste num lacrimejamento reflexo, causado por: ceratite, triquíase, distiquíase, conjuntivite, corpo estranho e também observar a posição, estrutura, refluxo e coloração dos pontos lacrimais.

Devemos salientar os seguintes testes:

- Teste do desaparecimento da fluoresceína ( Teste de Milder)

Este teste consiste na instilação de uma gota de fluoresceína a $2 \%$ no fundo de saco conjuntival do olho suspeito, e o menisco lacrimal é examinado com um filtro de cobalto da lâmpada de fenda após cinco minutos. A retenção do corante varia de 0 a $4+($ meniscos de 0 a $1+$ são considerados normais; menisco de $2+$ indica início de obstrução anatômica ou funcional; meniscos de 3 +a 4+ indicam alteração do fluxo lacrimal).

-Teste de Jones I

Este teste avalia a permeabilidade da via lacrimal excretora e consiste na instilação de fluoresceína a $2 \%$ no fundo de saco conjuntival do olho suspeito de obstrução e após 5 minutos, iremos notar este corante na ponta do algodão do cotonete que foi colocado no meato nasal inferior, previamente anestesiada a mucosa nasal com solução gel de xilocaína $2 \%$. Considera-se o teste positivo quando há o corante na ponta do cotonete, indicando via lacrimal pérvia.

-Teste de Jones II

É realizado logo após o Teste de Jones I. O paciente deverá estar sentado,instila-se colírio anestésico tópico na conjuntiva bulbar, dilata-se o ponto lacrimal inferior do olho suspeito de obstrução e irriga-se a via lacrimal com $2 \mathrm{ml}$ de soro fisiológico através do canalículo inferior, observa-se o soro que sai pela fossa nasal. O soro colorido significa uma obstrução na via lacrimal excretora baixa 


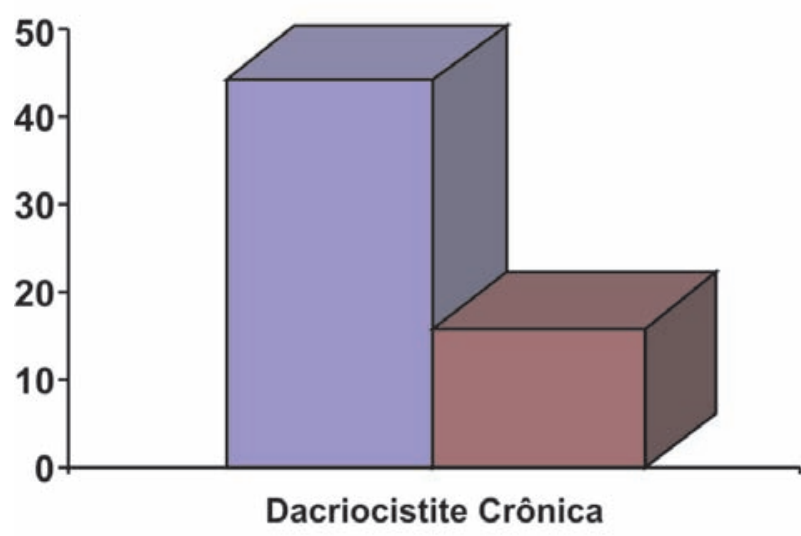

$\square$ feminino $\quad \square$ masculino

Figura 1: A relação de dacriocistite crônica com o sexo

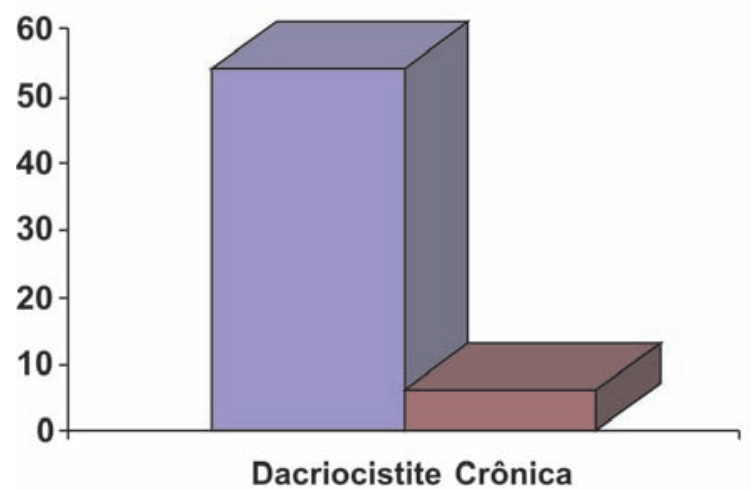

$\square$ raça branca $\square$ raça negra

Figura 3: Relação da dacriocistite crônica com a raça

\section{Resultados}

A média de idade dos pacientes com dacriocistite crônica atendidos no Setor de Vias Lacrimais da Universidade Federal de São Paulo foi de 55 anos, sendo $73 \%$ (44) do sexo feminino e $27 \%$ (16) do sexo masculino (Figura 1).

O envolvimento unilateral ocorreu em $95 \%$ (57) dos casos de dacriocistite crônica e somente 5\% (3) foram bilaterais. Neste trabalho, o acometimento do lado direito foi de $45 \%$ (26) e do lado esquerdo $55 \%$ (31) respectivamente (Figura 2)-.

Em relação à raça, prevaleceu a branca com $92 \%$

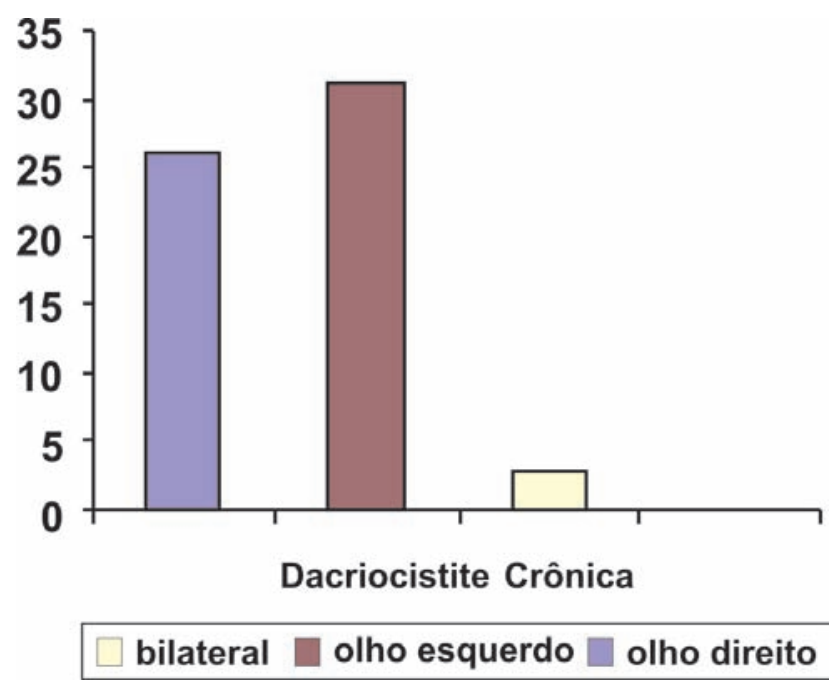

Figura 2: A relação da dacriocistite crônica com a lateralidade

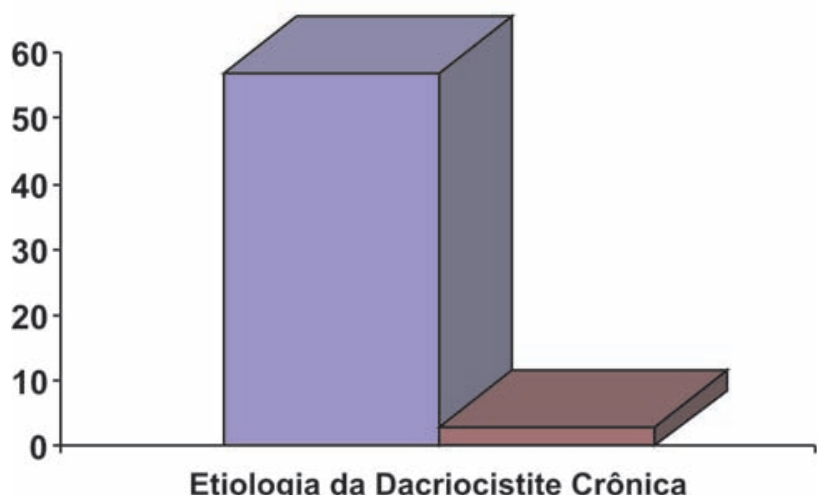

$\square$ idiopática $\square$ trauma

Figura 4: Etiologia da dacriocistite crônica

(54) dos casos dacriocistite crônica e 8\% (6 ) da raça negra (Figura 3).

A evolução para dacriocistite aguda ocorreu em $20 \%$ dos pacientes.

Em relação à etiologia da dacriocistite crônica, houve predominância da idiopática em 95\% (57) dos casos e somente 5\% (3) devido a trauma (Figura 4).

\section{Discussão}

Geralmente a frequência de dacriocistite crônica na população é de 1:50.000 pessoas $^{(10)}$.

A incidência da dacriocistite crônica é maior em 
caucasianos e rara nos negros, isto estaria relacionado com o diâmetro do canal ósseo do ducto nasolacrimal ${ }^{(11,12)}$, o que confirma os achados deste estudo.

As obstruções lacrimais são mais frequentes nas mulheres do que nos homens, segundo alguns autores, chegando a uma proporção de até $10: 1^{(13)}$, fato este que condiz com os resultados deste trabalho.

A dacriocistite é comumente unilateral, sendo bilateral um achado raro ${ }^{(14)}$, o que corrobora com os resultados deste trabalho.De acordo com a literatura, ambos os lados são igualmente envolvidos, uma vez que não há diferença significante no diâmetro ósseo de ambos os $\operatorname{lados}^{(14)}$.

$\mathrm{O}$ avanço da idade faz aumentar a incidência da dacriocistite crônica, devido ao processo involucional levando a estenose da ducto nasolacrimal, sendo comum na pós-menopausa, ao redor de 60 anos ou mais, perfazendo $74 \%$ dos casos, o que condiz com os achados desta pesquisa ${ }^{(15)}$.

\section{Conclusão}

A dacriocistite é mais frequente nas mulheres, rara na raça negra e apresenta predisposição hereditária, aumentando a incidência a partir da $6^{\mathrm{a}}$ década.

\section{RefERÊNCIAS}

1. Pérez Genovesi M. Patologia del aparato lacrimal. Arch Oftalmol B Aires. 1987;62(4):171-266.

2. Kushner BJ. Congenital nasolacrimal system obstruction. Arch Ophthalmol. 1982;100(4):597-600.

3. Nelson LR, Calhoun JH,Menduke H. Medical management of congenital nasolacrimal duct obstruction. Ophthalmology. 1985;92(9):1187-90.
4. Tucker N, Chow D, Stockl F, Codère F, Burnier M. Clinically suspected primary acquired nasolacrimal duct obstruction: clinicopathologic review of 150 patients. Ophthalmology. 1997;104(11):1882-6.

5. Bernardini FP, Moin H, Kersten RC, Reeves D, Kulwin DR. Routine histopathologic evaluation of the lacrimal sac during dacryocystorhinostomy: how useful is it? Ophthalmology. 2002;109(7):2434-5.

6. Altan-Yaycioglu R, Canan H, Sizmaz S, Bal N, Pelit A, Akova YA. Nasolacrimal duct obstruction: clinicopathologic analysis of 205 cases. Orbit 2010; 29(5):254-8.

7. Hendrickson DA, Cunningham RK, Veirs ER. Posttraumatic lacrimal-antral fistula: report of two cases. Ann Ophthalmol. 1977;9(4):457-6.

8. Garfin SW. Etiology of dacryocystitis and epiphora. Arch Ophthalmol. 1942;27:167-88.

9. Costa MN, Macchiaverni N. Dacriocistorrinostomia externa. Arq Bras Oftalmol. 1989:52(4):140.

10. Ivanisevic M, Bojic L, Lesin M, Zuljan I, Bucan K, Kovacic Z. Primary acquired nasolacrimal duct obstruction: epidemiological analysis of 91 patients. Med Jad. 2007;37(1-2):37-41.

11. Duke-Elder S, MacFaul PA. System of ophthalmology: the ocular adnexa. Saint Louis: Mosby; 1974. p. 7000. Vol 13. Part II.

12. Santos Fernández J. De la disposición anatómica del canal nasal em el negro que explica su menor predisposición a las afecciones de las vías lagrimales . Arch Oftal Hisp Am. 1903; 3:193-211.

13. Hurwitz JJ, Rutherford S. Computorized survey of lacrimal surgery patients. Ophthalmology. 1986;93(1):14-9.

14. Groessl SA, Sires BS, Lemke BN. An anatomical basis for primary acquired nasolacrimal duct obstruction. Arch Ophthalmol.1997;115(1):71-4. Erratum in Arch Ophthalmol. 1997;115(5):655.

15. Lee-Wing MW, Ashenhurst ME. Clinicopathologic analysis of 166 patients with primary acquired nasolacrimal duct obstruction. Ophthalmology. 2001;108(11):2038-40.

\section{Endereço para correspondência: \\ Silvia Helena Tavares Lorena \\ Rua Flórida, ${ }^{0} 1404$ - Brooklin \\ CEP 04561-030 - São Paulo (SP), Brasil \\ Tel: (11) 5507-2705}

E-mail: silvia.helenatavareslorena@yahoo.com.br 